\title{
Isolation and functional characterization of a cDNA coding a hydroxycinnamoyltransferase involved in phenylpropanoid biosynthesis in Cynara cardunculus $\mathbf{L}$ Cinzia Comino ${ }^{1}$, Sergio Lanteri ${ }^{1}$, Ezio Portis ${ }^{1}$, Alberto Acquadro1, Annalisa Romani ${ }^{2}$, Alain Hehn ${ }^{3}$, Romain Larbat ${ }^{3}$ and Frédéric Bourgaud ${ }^{* 3}$
}

Address: ${ }^{1}$ Di.Va.P.R.A. Plant Genetics and Breeding, University of Torino 10095, Grugliasco (Turin), Italy, ${ }^{2}$ Department of Pharmaceutical Sciences, University of Florence, 50019, Sesto Fiorentino (Florence), Italy and ${ }^{3}$ UMR 1121 INPL-INRA Agronomie Environnement, 54505 Vandoeuvre-lès-Nancy, France

Email: Cinzia Comino - cinzia.comino@unito.it; Sergio Lanteri - sergio.lanteri@unito.it; Ezio Portis - ezio.portis@unito.it; Alberto Acquadro - alberto.acquadro@unito.it; Annalisa Romani - annalisa.romani@unifi.it; Alain Hehn - alain.hehn@ensaia.inpl-nancy.fr; Romain Larbat - romain.larbat@ensaia.inpl-nancy.fr; Frédéric Bourgaud* - frederic.bourgaud@ensaia.inpl-nancy.fr

* Corresponding author

Published: 20 March 2007

BMC Plant Biology 2007, 7:14 doi:10.1186/147I-2229-7-14
Received: 21 November 2006

Accepted: 20 March 2007

This article is available from: http://www.biomedcentral.com/l47I-2229/7//4

(c) 2007 Comino et al; licensee BioMed Central Ltd.

This is an Open Access article distributed under the terms of the Creative Commons Attribution License (http://creativecommons.org/licenses/by/2.0), which permits unrestricted use, distribution, and reproduction in any medium, provided the original work is properly cited.

\begin{abstract}
Background: Cynara cardunculus L. is an edible plant of pharmaceutical interest, in particular with respect to the polyphenolic content of its leaves. It includes three taxa: globe artichoke, cultivated cardoon, and wild cardoon. The dominating phenolics are the di-caffeoylquinic acids (such as cynarin), which are largely restricted to Cynara species, along with their precursor, chlorogenic acid (CGA). The scope of this study is to better understand CGA synthesis in this plant.
\end{abstract}

Results: A gene sequence encoding a hydroxycinnamoyltransferase (HCT) involved in the synthesis of CGA, was identified. Isolation of the gene sequence was achieved by using a PCR strategy with degenerated primers targeted to conserved regions of orthologous HCT sequences available. We have isolated a 717 bp cDNA which shares $84 \%$ aminoacid identity and $92 \%$ similarity with a tobacco gene responsible for the biosynthesis of CGA from p-coumaroyl-CoA and quinic acid. In silico studies revealed the globe artichoke HCT sequence clustering with one of the main acyltransferase groups (i.e. anthranilate $\mathrm{N}$-hydroxycinnamoyl/benzoyltransferase). Heterologous expression of the full length HCT (GenBank accession DQ 104740) cDNA in E. coli demonstrated that the recombinant enzyme efficiently synthesizes both chlorogenic acid and $p$-coumaroyl quinate from quinic acid and caffeoyl-CoA or $p$-coumaroyl-CoA, respectively, confirming its identity as a hydroxycinnamoyl-CoA: quinate HCT. Variable levels of HCT expression were shown among wild and cultivated forms of $C$. cardunculus subspecies. The level of expression was correlated with CGA content.

Conclusion: The data support the predicted involvement of the Cynara cardunculus HCT in the biosynthesis of CGA before and/or after the hydroxylation step of hydroxycinnamoyl esters. 


\section{Background}

Cynara cardunculus L. is a perennial member of the Asteraceae family and has been sub-classified into three taxa: globe artichoke (var. scolymus L.), cultivated cardoon (var. altilis DC), and wild cardoon [var. sylvestris (Lamk) Fiori]. Molecular, cytogenetic and isozyme evidence suggests that wild cardoon is the ancestor of both cultivated forms [1-3].

Globe artichoke plays an important role in human nutrition, mainly in Mediterranean diet: immature inflorescences (capitula), commonly referred to as 'heads' or 'buds', are consumed as a fresh, canned or frozen vegetable, while more recently, demand has been driven by its reputation as health food.

The species has interesting applications in pharmacology [4]. The roots contain inulin, a natural fibre found to improve the balance of beneficial bacteria in the human gut, while the leaves and heads represent natural sources of bioactives such as luteolin and mono- and di-caffeoylquinic acids [5-8]. These compounds have been implicated in (i) the protection of proteins, lipids and DNA from oxidative damage caused by free radicals [9-11], (ii) the inhibition of cholesterol biosynthesis, contributing to the prevention of arteriosclerosis and other vascular disorders $[10,12,13]$, (iii) hepatoprotective, choleretic, diuretic and bile-expelling activities [4], (iv) the inhibition of HIV integrase, a key player in HIV replication and its insertion into host DNA $[14,15]$, and (v) antibacterial and antifungal activities [16-18]. All of these bioactivities have been attributed to the phenolics in the phenylpropanoid pathway [7], which is plant-specific [19]. The pathway catalyses the conversion of phenylalanine into a variety of hydroxycinnamic acids, which are the precursors for flavonoids, hydroxycinnamic acid conjugates and lignins [20]. Among phenolics, mono- and di-caffeoylquinic acids play a key-role in the overall anti-oxidant/health value of globe artichoke [8]. However, no information is available yet about the synthesis of these compounds in C. cardunculus.

This paper describes the cloning and biochemical characterization of an acyltransferase cDNA from globe artichoke. We explore the relationship between acyltransferases transcription and polyphenolic content in leaves, and establish a positive correlation between acyltransferase expression in various $C$. cardunculus accessions and polyphenolic content, especially CGA. Our observations suggest this acyltransferase is implicated in the biosynthesis of CGA and its derivatives.

\section{Results \\ Isolation of acyltransferase gene in globe artichoke} Using CODEHOP, targeting conserved regions of acyl transferase proteins (Fig. 1, black frames; Table 1), a 717 bp incomplete globe artichoke acyltransferase sequence was amplified. This sequence was extended towards both the 3'- and 5'-ends of the gene by RACE-PCR and a 1480 bp sequence was isolated. The resulting 1308 bp ORF (GenBank accession DQ104740) encodes a 436-residue protein (Fig. 1 in bold) with a predicted molecular weight of $\sim 50 \mathrm{kDa}$. Its closest in silico match is with a tobacco shikimate/quinate HCT [21], with which it shares $84 \%$ identity and $92 \%$ similarity; both proteins belong to a multifunctional superfamily of plant acyltransferases [22]. The globe artichoke acyltransferase has a histidinecontaining motif (HHAAD, aa 153-157, Fig. 1, gray boxes) identical to the highly conserved motif HXXXD which is characteristic for acyl transfer catalysis. A second consensus sequence, the DFGWG block found in other acyltransferases [22-24], is present from aa 382 to 386 (Fig. 1, gray boxes). The next most closely related sequence to the globe artichoke acyltransferase is an Arabidopsis thaliana HCT (80\% identity and 89\% similarity) (Fig. 2). More distantly related acyltransferases are those annotated as hydroxycinnamoyl-CoA quinate: hydroxycinnamoyl transferase (HQT) from tobacco and tomato [25].

\section{Heterologous expression of the identified acyltransferase}

To assess the activity of the globe artichoke isolated acyltransferase, the cDNA was cloned and heterologously expressed in E. coli, through the expression vector pET3a. The recombinant plasmid or the void pET3a vector (control) were introduced into E. coli BL21 (DE3) pLysE cells, and expression of soluble acyltransferase was first tested at standard induction temperature $\left(37^{\circ} \mathrm{C}\right.$ for $\left.8 \mathrm{~h}\right)$. SDSPAGE analysis indicated that the pellet fraction of recombinant bacteria contained an overexpressed protein with an apparent molecular mass of approximately $50 \mathrm{kDa}$, consistent with the expected mass for the translation product of the acyltransferase cDNA (Fig. 3). No acyltransferase protein could be detected by SDS-PAGE in the supernatant fraction of lysed recombinant cells. Reducing the temperature to $28^{\circ} \mathrm{C}$ during IPTG induction for 8 hours allows to increase the amount of soluble recombinant enzyme produced (Fig. 3) which can be detected in both fraction: pellet and supernatant. No corresponding protein is observed in samples prepared from bacteria carrying the control void vector.

\section{Enzyme assay}

The recombinant acyltransferase was used for substrate specificity studies. HPLC profiles were generated after incubation of the substrates caffeoyl-CoA or $p$-coumaroylCoA, with quinate or shikimate in the presence of recom- 


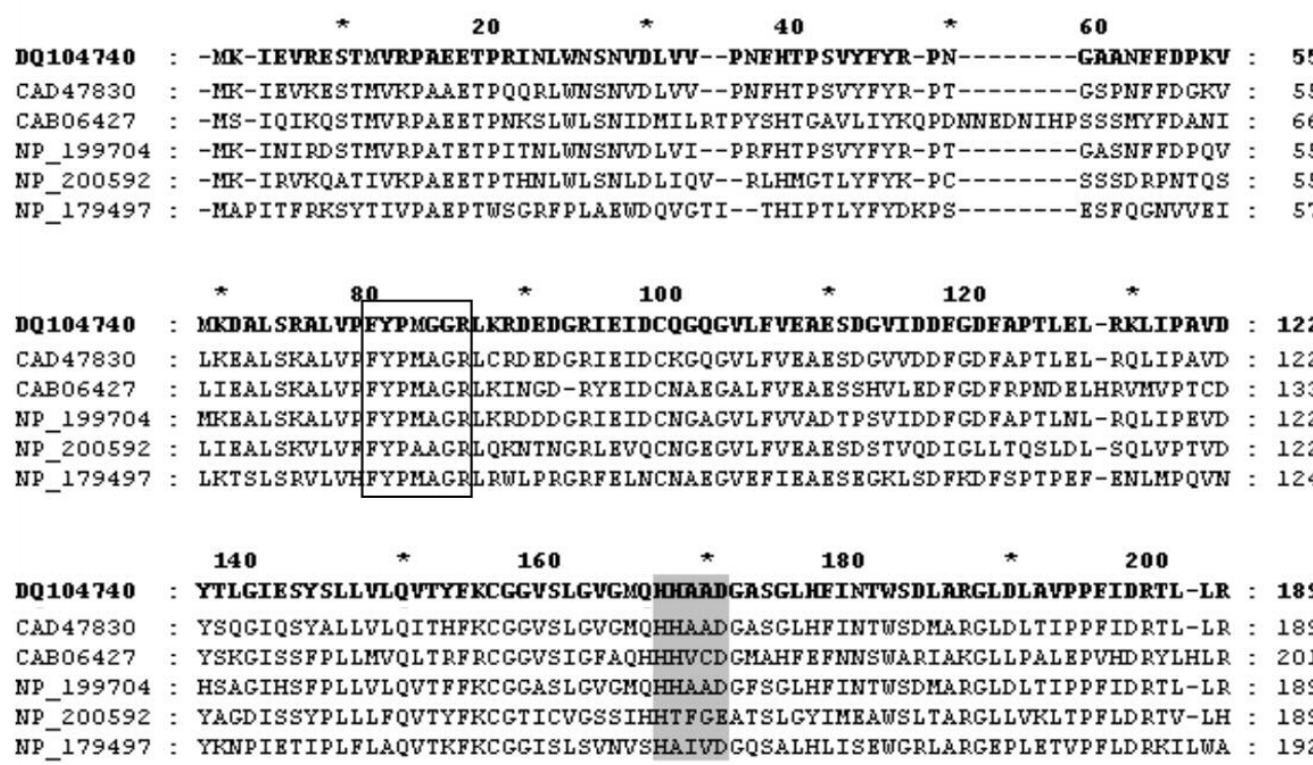

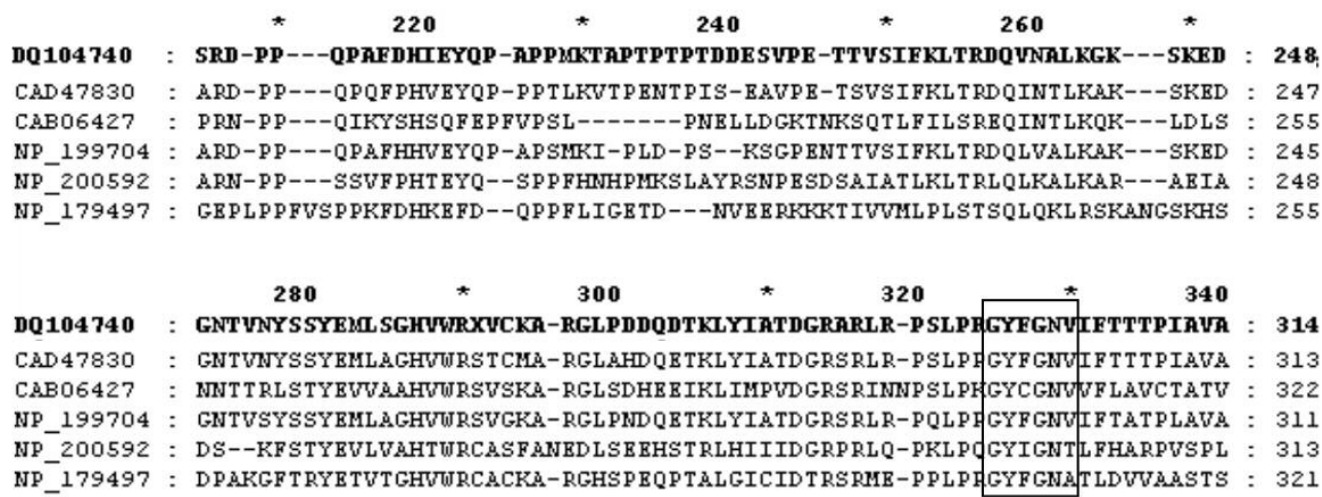

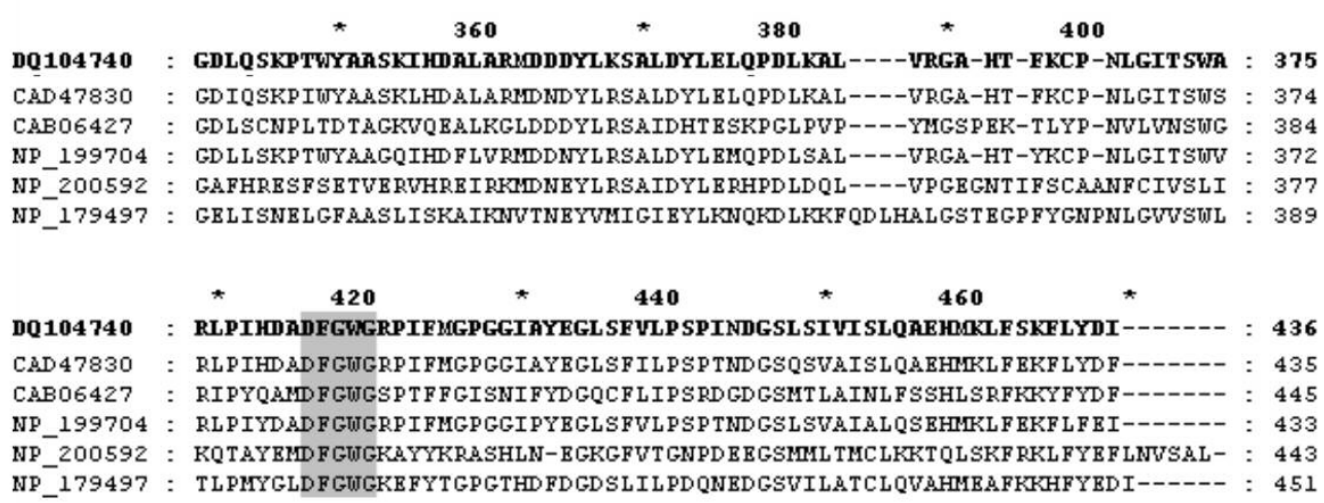

\section{Figure I}

Sequence alignment of HCT from artichoke with representative members of the plant hydroxycinnamoyl transferase family. CAD47830 from N. tabacum; NP_199704 from A. thaliana; CAB06427 from D. caryophyllus; NP_200592 from A. thaliana; NP_I79497 from A. thaliana; DQ 104740 (in bold) from C. cardunculus. Black frames indicate regions chosen to design CODEHOP; gray boxes indicate structural motifs conserved in the acyltransferase family. 


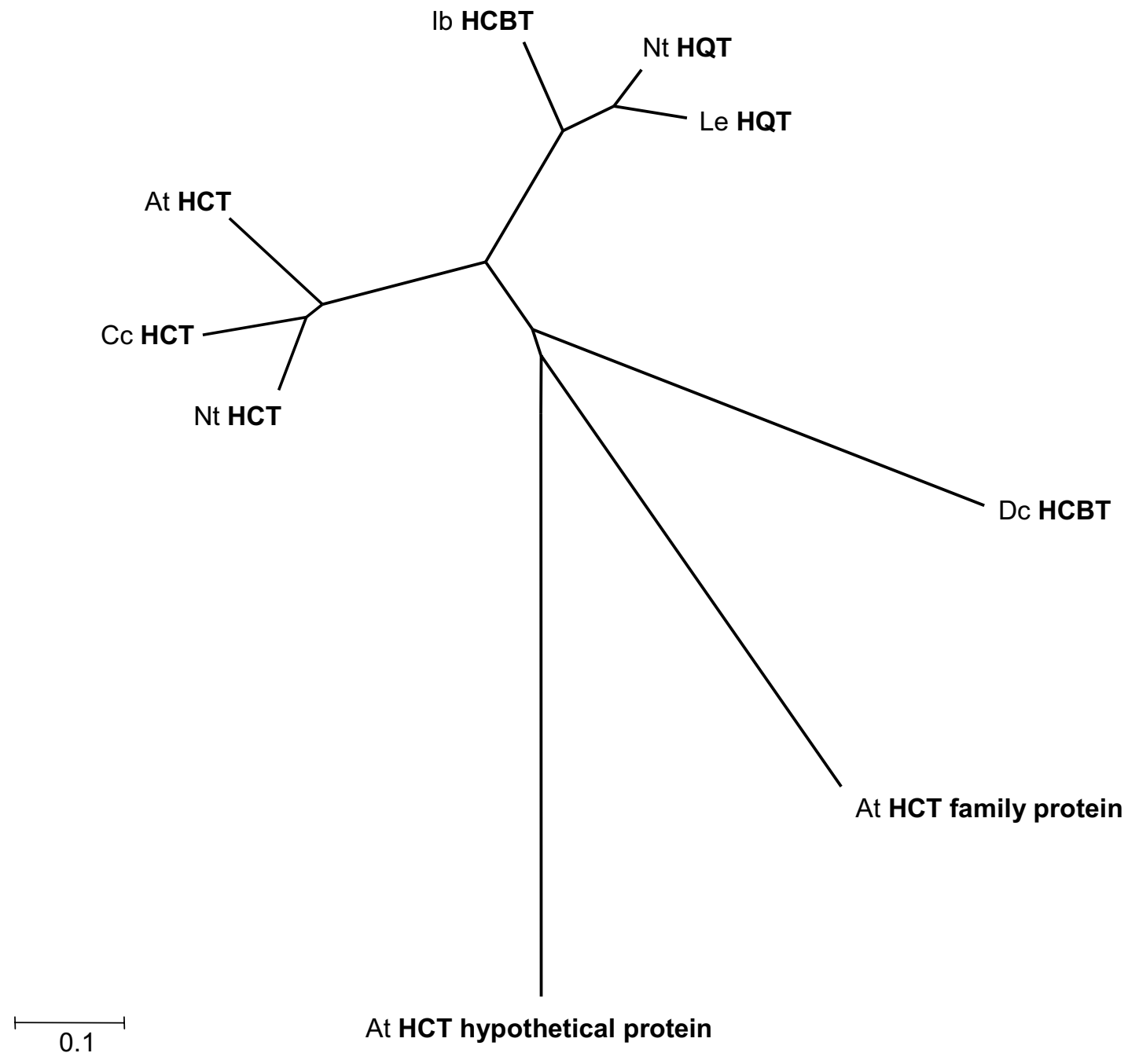

Figure 2

Phylogenetic analysis of acyltransferases. The tree was constructed by the neighbour-joining method. The length of the lines indicates the relative distances between nodes. Protein sequences used for the alignment are: DcHCBT, anthranilate Nhydroxycinnamoyl/benzoyltransferase of $D$. caryophyllus (Z84383); IbHCBT, N-hydroxycinnamoyl/benzoyltransferase from I. batatas (AB035I83); AtHCT, shikimate/quinate hydroxycinnamoyltransferase of A. thaliana (At5g48930); NtHCT, shikimate/ quinate hydroxycinnamoyltransferase of N. tabacum (AJ507825); NtHQT, hydroxycinnamoyl CoA quinate transferase of N. tabacum (CAE46932); LeHQT, hydroxycinnamoyl CoA quinate transferase of $L$. esculentum (CAE46933); At2G I 9070 and At5G57840, A. thaliana genes encoding putative acyltransferases; CcHCT, hydroxycinnamoyl CoA quinate transferase from $C$. cardunculus (DQ104740, this work).

binant bacteria crude extract carrying the pET-HCT or the void control vector. Both caffeoyl-CoA and p-coumaroylCoA were accepted as substrates, with either quinate and shikimate, to synthesize caffeoylquinate (i.e. chlorogenic acid), p-coumaroyl quinate, caffeoylshikimate or $p$-coumaroyl shikimate, depending on the substrates supplied. In presence of the recombinant proteins, a new product was detected in all cases (Fig. 4, grey lines); new peaks could not be detected when the reaction was performed with the control crude extract (black lines in Fig. 4). The reaction product was identified by comparing its retention time and its absorbance spectrum (200-400 nm, Fig. 5). We also successfully investigated the ability of the isolated acyltransferases to catalyse the reverse reaction (i.e. production of caffeoyl-CoA from chlorogenic acid), as described in other species $[21,26,27]$ : caffeoyl-CoA was 
Table I: Primer sequences used to isolate HCT gene in globe artichoke

\begin{tabular}{|c|c|}
\hline CODIFor & 5'-TTTTATCCNATGGCNGGDMG-3' \\
\hline CODIRev & 5'-AACGTTHCCRAARTANCC-3' \\
\hline ART2For & 5'-ATGGCAACACTGTCAATTA-3' \\
\hline ART2For-nested & 5'-CCCGACGATCAGGATA-3' \\
\hline ART2Rev & 5'-ACCGCCGGGATGAGTT-3' \\
\hline ART2Rev-nested & 5'-CCGCCTCCACGAACAA-3' \\
\hline UTR5' & 5'-TTCCGTTTCGTTTCTTCAA-3' \\
\hline UTR3' & 5'-TGGCCATAACCATTTTAGATAT-3' \\
\hline HCTFor & 5'-GGGTTTCATATGAAGATCGAGGTGAGAGAA-3' \\
\hline HCTRev & 5'-CGGGATCCTTAGATATCATATAGGAACTTGC-3' \\
\hline ART3For & 5'-TCCCCAATTTTCACACAC-3' \\
\hline ART3Rev & 5'-AAGTGCCGATTTTAGATAAT-3' \\
\hline
\end{tabular}

detected when the chlorogenate was incubated with CoEnzyme A in presence of the recombinant protein (Fig. $4 \mathrm{~b})$.

\section{Determination of kinetic parameters}

Kinetic parameters of the HCT enzyme were evaluated for the different substrates (Table 2). The affinity of the enzyme for quinate as acceptor was higher than for shikimate. Moreover $p$-coumaroyl-CoA was the most efficient donor, with a $V_{\text {max }} / K_{\mathrm{m}}$ of 0.041 , followed by caffeoyl-CoA, with a value of 0.01 .

\section{Identification and quantification of polyphenolics}

Six compounds belonging to quinic acid esters were quantified (Table 3, see Fig. 6 for chromatograms). 'Violet Margot' and wild cardoon leaves presented a high mean content of total caffeoylquinic acid (respectively, 60.8 \pm 1.98 and $59.9 \pm 4.30 \mathrm{mg} / \mathrm{g}$ dry matter) while the cultivated cardoon and 'Romanesco C3' leaves showed lower contents $(17.1 \pm 2.59$ and $15.0 \pm 3.10 \mathrm{mg} / \mathrm{g}$ dry matter $)$. Chlorogenic acid was the most abundant compound among quinic acid esters in globe artichoke (both 'Romanesco C3' and 'Violet Margot') and cultivated cardoon, but was less represented than di-caffeoylquinic acids in wild cardoon. All samples contained very low levels of $p$ coumaroylquinic acid.

\section{Northern blot analysis}

A northern blot approach was taken to identify a possible correlation between HCT expression and polyphenolics/ chlorogenic acid content. As the different plant species probably not exhibit exactly the same HCT sequence, PCR was performed on each genomic DNA in order to isolate a highly specific HCT probe (probe 1 from globe artichoke, probe 2 from cultivated cardoon, and probe 3 from wild cardoon). A sequence alignment established a high level of similarity (99\%) between probe 2 and 3 (respectively isolated from cultivated cardoon and wild cardoon), but a rather lower level (80\%) between probes 2 (or 3) and probe 1, from globe artichoke (data not reported). When 'Romanesco C3' and 'Violet Margot' RNA were probed with the globe artichoke HCT sequence

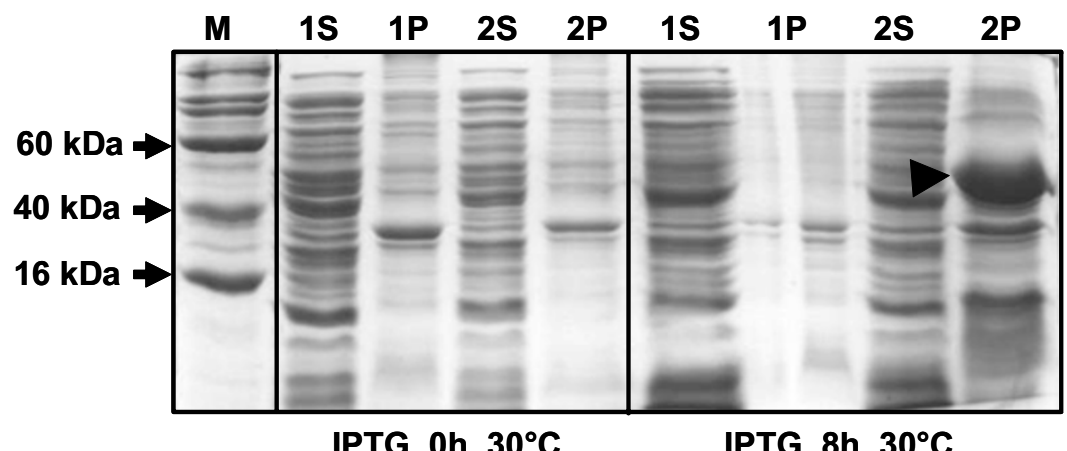

IPTG $0 \mathrm{~h} 30^{\circ} \mathrm{C}$

IPTG $8 \mathrm{~h} 30^{\circ} \mathrm{C}$

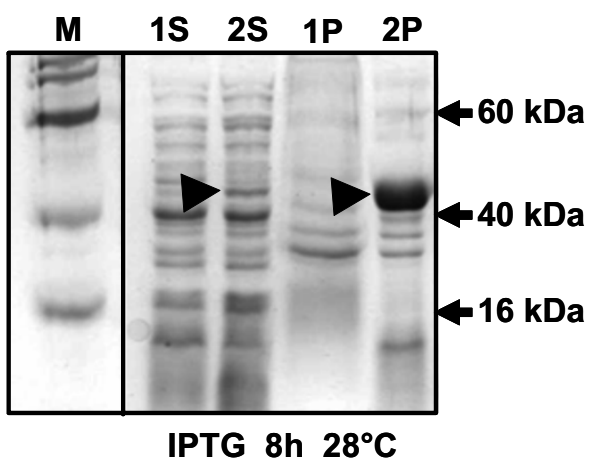

\section{Figure 3}

Expression of recombinant HCT in E. coli. Protein content of non-induced $(T=0 \mathrm{~h})$ and induced $(\mathrm{T}=8 \mathrm{~h})$ non-transformed cells $(\mathrm{I})$ were compared with those of non-induced $(T=0 \mathrm{~h})$ and induced $(\mathrm{T}=8 \mathrm{~h})$ transformed cells $(2)$. After induction at $37^{\circ} \mathrm{C}$ for $8 \mathrm{~h}, \mathrm{HCT}$ protein cannot be detected in the soluble fraction $(2 \mathrm{~S}$ at $\mathrm{T}=8 \mathrm{~h})$, but is present in the soluble fraction after induction at $28^{\circ} \mathrm{C}$. Arrows indicate the $\sim 50 \mathrm{kDa} H C T$ protein. Molecular marker masses indicated on the left. (I = empty vector; 2 = vector $-\mathrm{HCT} ; \mathrm{S}=$ soluble fraction; $\mathrm{P}=$ pellet; $\mathrm{M}=$ molecular weight marker). 

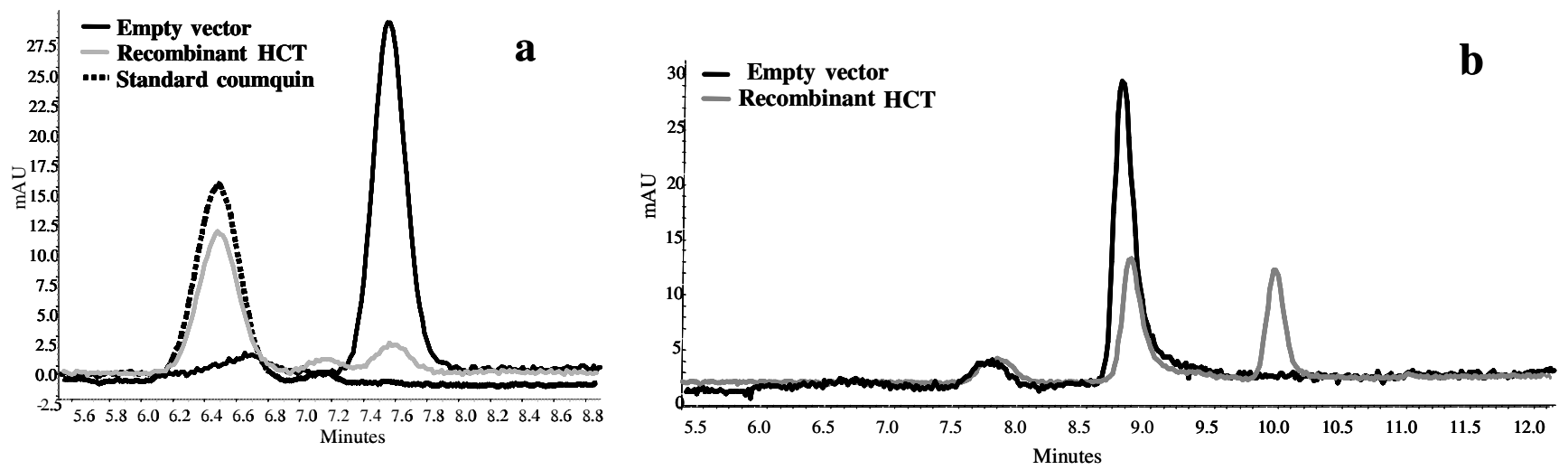

Figure 4

HPLC analysis of the HCT reaction products. An aliquot of the incubation reaction without (black line) or with (gray line) recombinant HCT was analysed. (a) HCT reaction with $p$-coumaroyl-CoA and quinate; standard of $p$-coumaroyl-quinate (dotted line) is used as reaction control; (b) HCT reverse reaction with chlorogenic acid and CoA.

(probe 1), the latter showed a higher transcript abundance in its leaves than the former (Fig. 7). Moreover, northern blot on cultivated and wild cardoon RNAs challenged with either probe 2 and 3, showing that a higher level of HCT transcript was present in the wild form (Fig. $7)$. Results are summarized in table 3.

\section{Discussion}

Phenolic compounds are by far the commonest of plant therapeutic molecules [28], and the major species present in globe artichoke leaves are the di-caffeoylquinic acids (e.g. cynarin), and their precursor CGA, a soluble phenolic which is widespread throughout the plant kingdom. The definition of the CGA biosynthetic pathway remains controversial, with three alternative routes (Fig. 8) under current consideration [25]. These are (1) CGA synthesis using a caffeoyl-glucoside as the active intermediate; (2) synthesis of CGA from caffeoyl-CoA and quinic acid by means of HQT (hydroxycinnamoyl-CoA: quinate HCT), which differs from HCT in its preference for quinate over shikimate as a substrate; and (3) synthesis of $p$-coumaroyl-quinate by HCT or HQT and its subsequent hydroxylation by $p$-coumarate-3'-hydroxylase $\left(\mathrm{C}^{\prime} \mathrm{H}\right)$ to form CGA. The first route has been identified in sweet potato by Villegas and Kojima [29], who were able to purify hydroxycinnamoyl D-glucose:quinate HCT and show that caffeoyl D-glucose and quinic acid are the substrates for the biosynthesis of CGA. Routes (2) and (3) were unequivocally established by Ulbrich and Zenk in several differentiated plants and undifferentiated cell suspension cultures [27].

Recently, both the second and third CGA synthesis routes have received experimental support. The biochemical characterization of $\mathrm{C}^{\prime} \mathrm{H}[30,31]$ and hydroxycinnamoyl-
CoA transferase HCT [21] suggests that CGA can be synthesized via the third route. However, since both HCT and $\mathrm{C}^{\prime} \mathrm{H}$ are active in A. thaliana, a species which does not accumulate CGA, it is unlikely that this route can be generally exploited by plants which accumulate significant amounts of CGA [25]. In tomato, it was difficult to establish whether HQT acts directly on caffeoyl-CoA and quinic acid to produce CGA, or whether it synthesizes $p$-coumaroyl quinate from $p$-coumaroyl-CoA and quinic acid, which is subsequently converted to CGA by the activity of $\mathrm{C}^{\prime} \mathrm{H}$ [25]. The second route was assumed to be dependent on the relative sizes of the caffeoyl-CoA and p-coumaroyl-CoA pools present. Nevertheless, strong support for the prevalence of the second route, at least in tomato, was provided by experiments in which the silencing of HQT caused the level of leaf CGA to fall by $98 \%$, and to rise by $85 \%$ when it was over-expressed.

In a study of the phenolic content in various globe artichoke tissues and organs, total phenol concentration was shown to be greatest in the leaves, and declined in the heads during their development [8]. The variation in antioxidant activity (generated by phenolic compounds) in globe artichoke extracts may, therefore, be attributed to the choice of plant tissue used as the source of extract, rather than to any variation in genotype or environment. Thus we used leaf as our source of mRNA in order to generate the necessary CDNA, and exploited CODEHOP to isolate globe artichoke HCT. The heterologous (in E. coli) expression product of the cloned HCT sequence was a $\sim 50$ $\mathrm{kDa}$ recombinant protein, which was active when provided with either $p$-coumaroyl-CoA or caffeoyl-CoA ester as acyl donors, at comparable $K_{\mathrm{m}}$ values of $53.0 \pm 13.0 \mu \mathrm{M}$ and $61.7 \pm 0.004 \mu \mathrm{M}$, respectively. Moreover, the artichoke HCT showed a preference for quinic acid over 


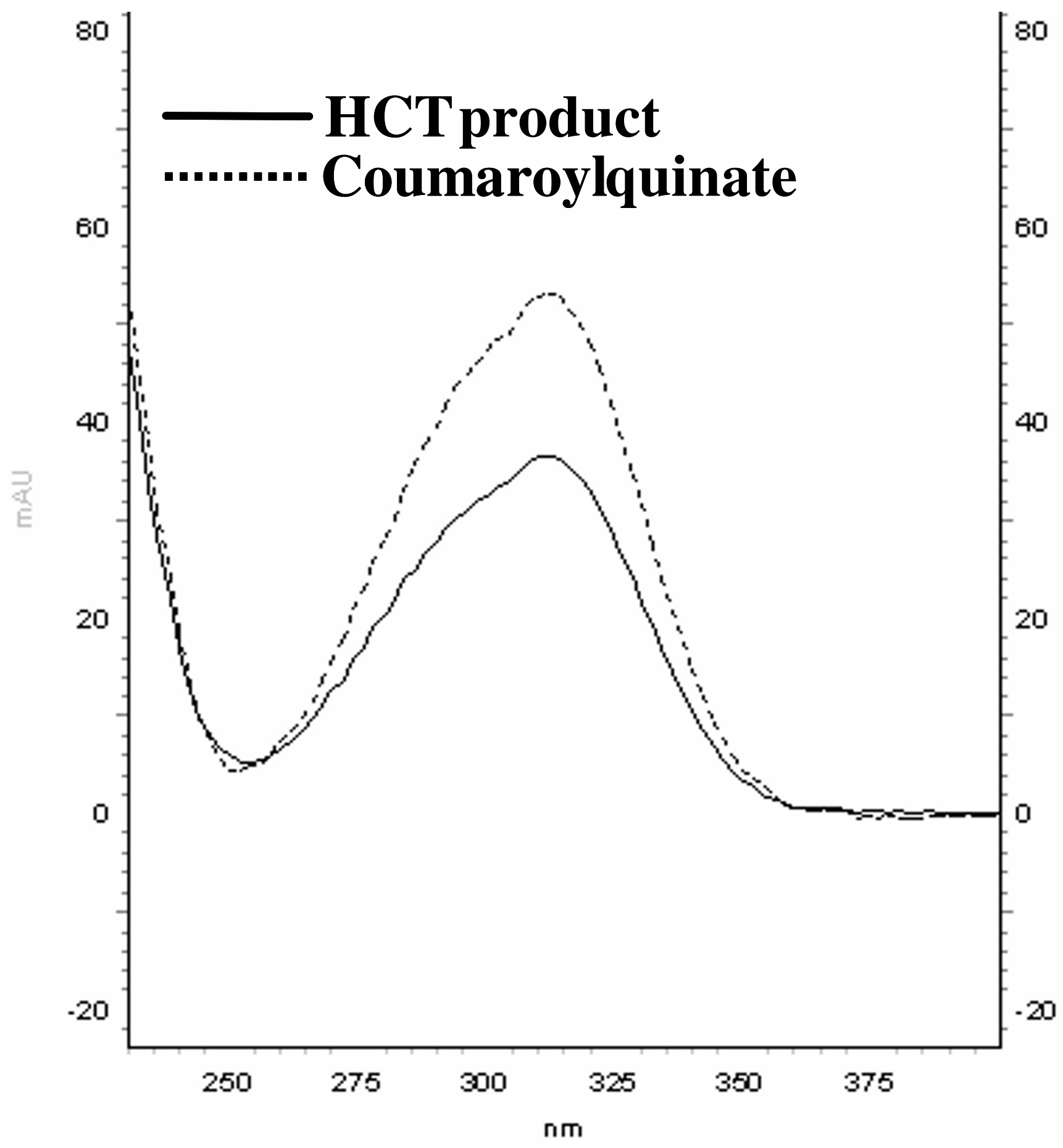

Figure 5

Example of comparison between absorption spectrum of the reaction product and authentic standard. Absorption spectra of $p$-coumaroyl-quinate: standard (dotted line) and product by reaction with HCT (black line).

shikimic acid as an acceptor $(53.0 \pm 13.0 \mu \mathrm{M} v$ s $701.7 \pm$ $52.0 \mu \mathrm{M})$. This behaviour contrasts with that of tobacco HCT [21], but is consistent with the activity of HQT isolated from tobacco and tomato [25]. Interestingly, although the globe artichoke HCT sequence is closely related to that of its tobacco ortholog, its activity appears to be more similar to that of tobacco and tomato HQT. 
Table 2: Kinetic parameters of recombinant HCT

\begin{tabular}{llccc}
\hline Varying substrate & Saturating substrate & \multicolumn{2}{c}{ kinetic parameters } \\
\cline { 3 - 4 } & & $K_{\mathbf{m}}(\mu \mathrm{M})$ & $\boldsymbol{V}_{\max }(\mathrm{nkat} / \mathrm{mg})$ & $\boldsymbol{V}_{\text {max }} / K_{\mathbf{m}}(\mathrm{nkat} / \mathrm{mg} / \mu \mathrm{M})$ \\
\hline quinate & p-coumaroyl-CoA & $9.2 \times 10^{3} \pm 5.6 \times 10^{3}$ & $2.1 \pm 1.2$ & 0.0002 \\
p-coumaroyl-CoA & quinate & $53.0 \pm 13.0$ & $2.2 \pm 0.5$ & 0.041 \\
p-coumaroyl-CoA & shikimate & $701.67 \pm 52$ & $2.06 \pm 0.55$ & 0.003 \\
Caffeoyl-CoA & quinate & $61.75 \pm 0.004$ & $0.67 \pm 0.04$ & 0.01 \\
\hline
\end{tabular}

The $K_{\mathrm{m}}$ and $V_{\max }$ values were calculated from triplicates by the Lineweaver-Burk method.

In order to evaluate the role of HCT in the biosynthetic pathway of CGA in globe artichoke, the purified enzyme was provided in vitro with quinic acid and either $p$-coumaroyl-CoA or caffeoyl-CoA. Since the enzyme was active with both $p$-coumaroyl-CoA and caffeoyl-CoA, it is clear that this HCT can act either before and/or after 3'-hydroxylation step. Other experiments have demonstrated that the heterologously expressed HCT, in the presence of quinic acid as the acyl donor, is four times more efficient when provided with $p$-coumaroyl-CoA rather than with caffeoyl-CoA ( $V_{\max } / K_{\mathrm{m}}$ values of, respectively, 0.041 and $0.01)$. However, these observations do not constitute an absolute proof that the third biosynthetic route is preferred over the second, since the level of HCT is not necessarily limiting in vivo. Note that CGA synthesis is also regulated by its interaction with $\mathrm{C}^{\prime} \mathrm{H}$, a $\mathrm{P} 450$ whose enzymatic turnover was found to be low [32].

Globe artichoke HCT belongs to a versatile plant acyltransferase family that shares certain structural motifs (Fig. 1, grey boxes), including several plant members involved in a number of secondary metabolism pathways. When the sequence alignment of the acyltransferase family was used to construct a phylogenetic tree (Fig. 2), the globe artichoke HCT was found to cluster with the major anthranilate N-hydroxycinnamoyl/benzoyltransferase group defined by Burhenne et al. [33]. It is clearly closely related to its tobacco and $A$. thaliana orthologs.

C. cardunculus includes two crop species, the globe artichoke and the cultivated cardoon, along with the ancestral wild cardoon. In our samples, $p$-coumaroylquinic acid was ubiquitously detected at a low concentration (Table $3)$, presumably because this quinate ester is a transient intermediate, unlike chlorogenic acid, which is considered to be an accumulation product in several plant species [30]. Di-caffeoylquinic acid synthesis remains unknown in higher plants. However, due to their close structural relationship with CGA, it is reasonable to suppose that the di-caffeoylquinic acids are derived from simple quinic acid monoesters. CGA and di-caffeoylquinic acid quantification studies on the leaves of four plant accessions were carried out to identify any correlations between these two families of molecule (Table 3). The globe artichoke 'Violet Margot' and the cultivated cardoon contained comparable levels of CGA and di-caffeoylquinic acids. On the other hand, in the globe artichoke 'Romanesco C3' there was ten fold more CGA than di-

Table 3: Caffeoylquinic acids content in leaves of globe artichoke, cultivated and wild cardoon and expression level of HCT in the different plant accessions

\begin{tabular}{|c|c|c|c|c|}
\hline & $\begin{array}{l}\text { Globe artichoke } \\
\text { 'Romanesco C3' }\end{array}$ & $\begin{array}{l}\text { Globe artichoke 'Violet } \\
\text { Margot' }\end{array}$ & Cultivated cardoon & Wild cardoon \\
\hline Caffeoylquinic acid (I)* & $0.21 \pm 0.06 c$ & $2.01 \pm 0.03 \mathrm{a}$ & $0.6 \mathrm{I} \pm 0.07 \mathrm{~b}$ & $1.83 \pm 0.03 \mathrm{a}$ \\
\hline Chlorogenic acid (5-CQA) (2) & $13.49 \pm 2.73 b c$ & $32.74 \pm 0.79 a$ & $9.32 \pm 1.18 \mathrm{c}$ & $19.02 \pm 0.97 b$ \\
\hline$p$-Coumaroylquinic acid (3) & $0.28 \pm 0.03 \mathrm{ab}$ & trace & $0.18 \pm 0.03 b$ & $0.35 \pm 0.03 a$ \\
\hline Feruloylquinic acid (4) & $0.96 \pm 0.32 \mathrm{a}$ & $0.79 \pm 0.06 \mathrm{a}$ & $0.13 \pm 0.00 c$ & $0.34 \pm 0.11 \mathrm{~b}$ \\
\hline Dicaffeoilquinic acids $(5,6)$ & $1.33 \pm 0.66 \mathrm{~d}$ & $26.03 \pm 1.09 b$ & $7.16 \pm 1.30 \mathrm{c}$ & $39.04 \pm 3.45 \mathrm{a}$ \\
\hline Total Caffeoylquinic acids $(1,2,5,6)$ & $15.03 \pm 3.10$ & $60.78 \pm 1.98$ & $17.09 \pm 2.59$ & $59.89 \pm 4.30$ \\
\hline HCT expression & $+/-$ & + & $+/-$ & + \\
\hline
\end{tabular}

Caffeoylquinic concentrations are expressed in $\mathrm{mg} / \mathrm{g}$ dry matter. Within a column, means with the letter are not significantly different $(\mathrm{P}<0.0 \mathrm{l}$; Tukey's HSD test).

HCT expression was measured by northern blot analysis achieved with HCT probes specifically designed for each plant accession. $+/-$ indicates a barely detectable signal, + indicates a strong signal.

* Numbers in brackets refer to peaks reported in Fig. 6 

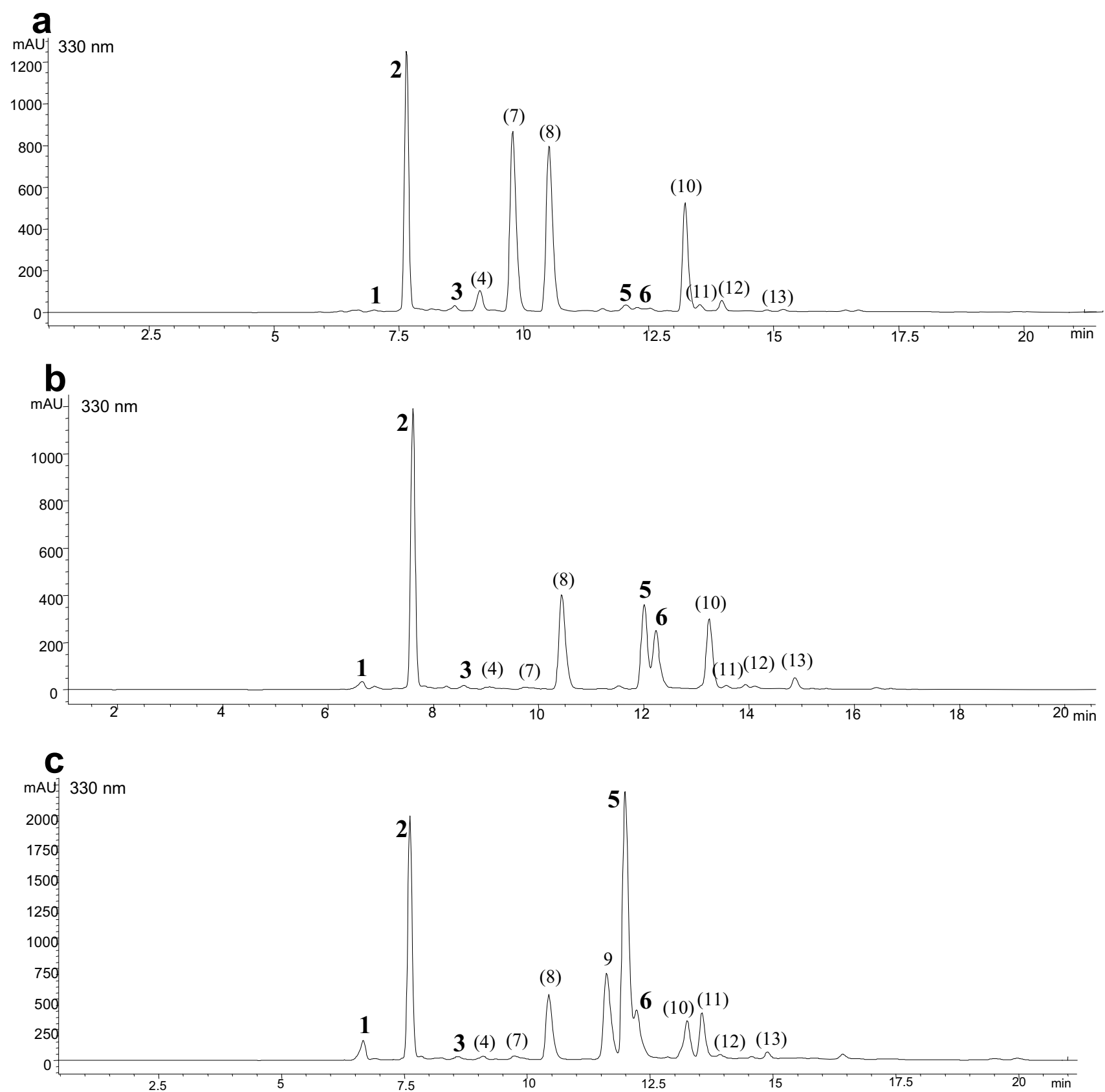

Figure 6

HPLC/DAD profiles at $330 \mathrm{~nm}$ of the compounds identified in C. cardunculus. (a) globe artichoke, (b) cultivated cardoon, and (c) wild cardoon. Peaks: I. caffeoylquinic acid; 2. chlorogenic acid (5-CQA); 3. p-coumaroylquinic acid; 4. feruloylquinic acid; 5. and 6. di-caffeoylquinic acids; 7. luteolin 7-O-rutinoside; 8. luteolin 7-O-glucoside; 9. luteolin 7-O-glucuronide; 10. luteolin malonylglucoside; II. apigenin 7-O-glucuronide; I2. luteolin; I3. apigenin. In bold are indicated the caffeoylquinic acids and in brackets others compounds detected in C.cardunculus.

caffeoylquinic acids, while in the wild cardoon, this difference was about two fold. Therefore, the regulation of the synthesis of di-caffeoylquinic acids should be, possibly, genotype-dependent.
Northern blots, using cDNA from the same three C. cardunculus subspecies analysed above, were performed to study patterns of HCT expression. As these diverse genotypes could carry distinct allelic forms of HCT, we devel- 

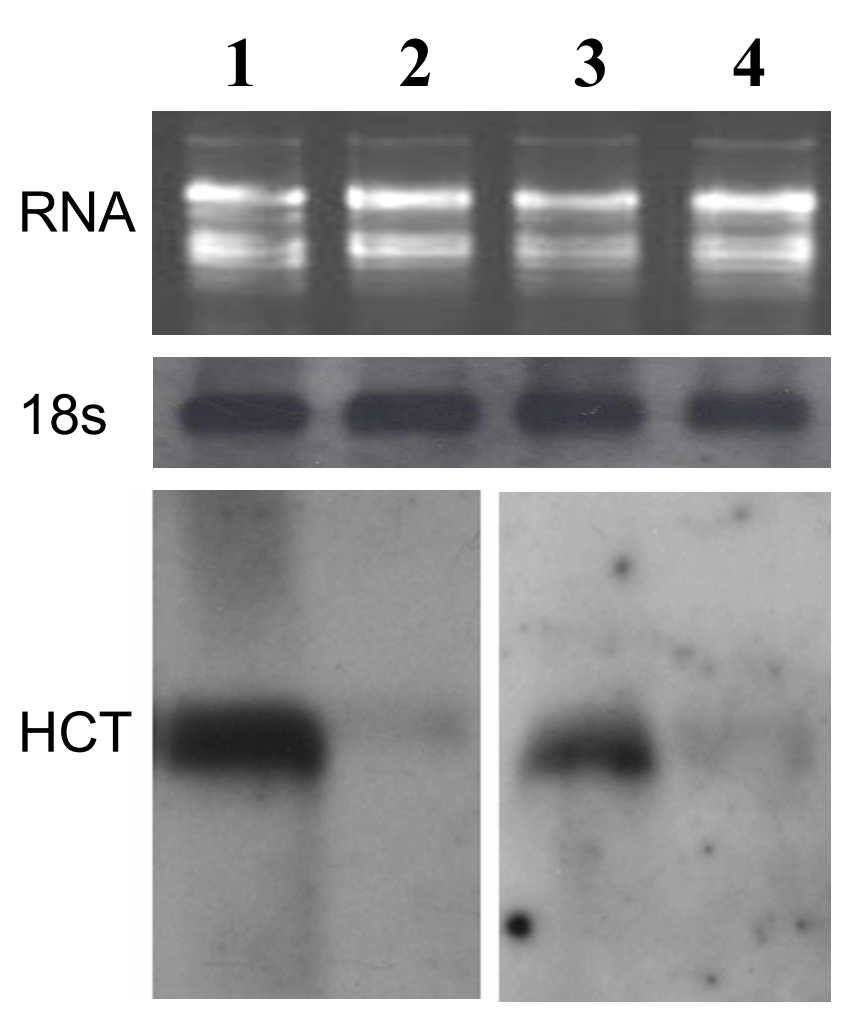

Figure 7

Northern blot analyses for HCT expression. First and second panels are, respectively, total RNA ethidium bromide-stained prior to membrane transfer and I8S expression to control RNA quality and sample loading. Third panel is the HCT expression with probel in 'Violet Margot' (I), Romanesco C3' (2), and with probe 2 in wild cardoon (3) and cultivated cardoon (4).

oped three species-specific probes [probes 1 (globe artichoke), 2 (cultivated cardoon) and 3 (wild cardoon). A positive relationship between the quantity of HCT transcript and the content of caffeoylquinic acids was observed in all accessions. Since HCT silencing induces an increase (or no significant change) in the amount of caffeoylquinic compounds in tobacco [34], whereas HQT silencing (in tomato) results in a decrease in CGA content [25], HQT transcripts may well play a pivotal role in determining the make-up of the CGA pool, and the behaviour of HCT in globe artichoke is fully consistent with this model.

\section{Conclusion}

CGA is particularly abundant in species belonging to the Asteracaeae, Solanaceae and Rubiaceae families [35], but its mode of biosynthesis is still unclear in many plants. We have described the cloning and expression of HCT, an acyltransferase acting both upstream and downstream of the 3'-hydroxylation step. In addition, for both wild and cultivated forms of C. cardunculus, the expression of HCT appears to be correlated with leaf polyphenolic content, especially with respect to caffeoylquinic acid derivatives, suggesting that this HCT has an essential role in the synthesis of CGA and related esters.

In a recent report [25], caffeoyl-CoA has been firmly established as a major substrate for the acylation of quinic acid and the synthesis of CGA in Solanaceous plants. Our future research activity will be focused in analysing the in vivo expression of HCT, as well as on the isolation of other acyltransferases, such as HQT, which may be involved in the phenylpropanoid pathway of C. cardunculus

\section{Methods}

Plant material and RNA extraction

Leaves of globe artichoke, cultivated cardoon and wild cardoon were collected from experimental fields at the University of Catania in Cassibile, Sicily (Italy). Total RNA was extracted from approximately $100 \mathrm{mg}$ fresh tissue using the "RNAwiz" reagent (Ambion, USA), following the manufacturer's instructions. Final RNA concentration was determined by spectrophotometry, and its integrity was assessed by electrophoresis in $1 \%(\mathrm{w} / \mathrm{v})$ formaldehyde-agarose gel [36].

\section{Purification and cloning of globe artichoke HCT}

Reverse transcription from total RNA was achieved using poly(dT)primer and M-MuLV RNaseH- RT (Finnzymes, Finland), following the manufacturer's instructions. Incomplete cDNAs were derived by PCR, using as template the cDNA generated by reverse transcription. Based on conserved regions of the acyltransferase amino acid sequence (Fig. 1), primers COD1For and COD1Rev (Table 1 ) were designed, with each primer consisting of a short 3' degenerate core and a longer 5' consensus clamp region. As recommended by Morant et al. [37], a cDNA amplification step was first performed, and the fragment of expected size was isolated from $1 \%$ agarose gel separations of the total amplicon. DNA sequences were resolved by BMR genomics [38]. Specific primers were designed for 3'- and 5'-end amplification of the HCT transcript, based on the derived incomplete cDNA sequence (Table 1). For the 3'-end, the template was the poly $(\mathrm{dT})$ reverse transcription product, and the primers consisted of poly(dT) oligonucleotides in combination with the specific primers ART2For and ART2For-nested (Table 1). The fragment of expected size was isolated from an agarose gel separation, cloned into $\mathrm{pCR}^{\circledR} 2.1$ (Invitrogen, USA), and sequenced. For the 5'-end, full-length cDNA produced with the SuperScript $^{\mathrm{TM}}$ Plasmid System (Invitrogen, USA) was inserted into the pCMV•SPORT6 plasmid. PCR was performed on this cDNA library using the antisense primer ART2Rev and ART2Rev-nested (Table 1), along with the universal SP6 specific primer. The expected fragment was isolated from 


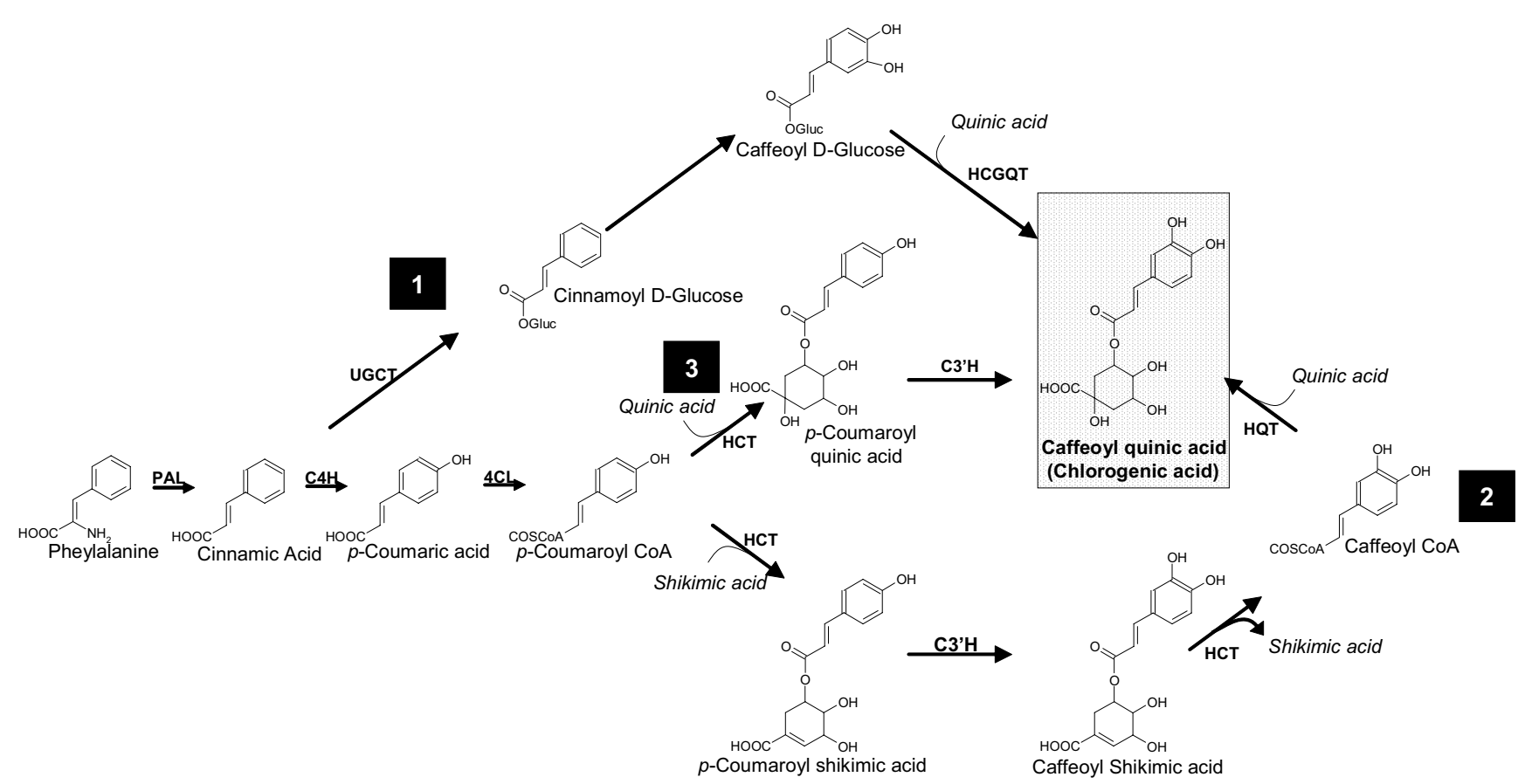

Figure 8

The three routes in phenylpropanoid metabolism (labelled I, 2 and 3) proposed for the chlorogenic acid synthesis in plants. Enzymes involved in the pathway are: $\mathrm{PAL}$, phenylalanine ammonia lyase; $\mathrm{C} 4 \mathrm{H}$, cinnamate 4-hydroxylase; 4CL, 4-hydroxycinnamoyl CoA ligase; $\mathrm{HCT}$, hydroxycinnamoyl CoA shikimate/quinate hydroxycinnamoyl transferase; $\mathrm{C} 3 \mathrm{H}$, $\mathrm{p}-$ coumarate 3'-hydroxylase; HQT, hydroxycinnamoyl CoA quinate hydroxycinnamoyl transferase; UGCT, UDP glucose: cinnamate glucosyl transferase; HCGQT, hydroxycinnamoyl D-glucose: quinate hydroxycinnamoyl transferase (adapted from [25]).

an agarose gel separation, cloned into $\mathrm{pCR}^{\circledR} 2.1$ (Invitrogen, USA) and sequenced. The full length sequence was validated by an amplification using primers UTR5' and UTR3' (Table 1), designed from the sequence of the 5'and 3 '-ends of the HCT transcript. Multiple sequence alignments were performed using ClustalW [39], with standard parameters. Phylogenetic analysis was conducted using MEGA version 3.0 [40].

\section{Heterologous expression in E. coli}

The HCT open reading frame (ORF) was amplified using HCTFor and HCTRev primers (Table 1), which additional recognition sites NdeI (5'-end) and BamHI (3'-end). The amplified fragment was restricted with NdeI and BamHI, and ligated into the cloning site of digested pET3a plasmid (Novagen, USA). The resulting recombinant pET3aHCT plasmid was transformed into E. coli strain BL21(DE)pLysE, and grown on a selective medium (LB, Luria-Bertani, broth agar with $50 \mathrm{mg} / \mathrm{l}$ ampicillin and 34 $\mathrm{mg} / \mathrm{l}$ chloramphenicol). Individual colonies were transferred to $4 \mathrm{ml}$ overnight cultures in the same medium, and $2 \mathrm{ml}$ of bacterial cell suspensions were then grown for $3 \mathrm{~h}$ at $28^{\circ} \mathrm{C}$ in $50 \mathrm{ml}$ of LB liquid medium with shaking $(200$ $\mathrm{rpm}$ ). Isopropyl- $\beta$-D-thiogalactopyranoside (IPTG) was added to a final concentration of $1 \mathrm{mM}$ and the cultures were grown for further $8 \mathrm{~h}$ at $28^{\circ} \mathrm{C}$. After centrifugation for $10 \mathrm{~min}$ at $5000 \mathrm{~g}$, the pellet was resuspended in $1 \mathrm{ml}$ of phosphate-buffered saline pH 7.5 and lysed by three cycles of freezing (in liquid nitrogen) and thawing (at $37^{\circ} \mathrm{C}$ ), followed by three bursts of $30 \mathrm{~s}$ sonication on ice. Sonicated cells were centrifuged at $4^{\circ} \mathrm{C}$ and $14,000 \mathrm{~g}$ for 5 min, and both supernatant and pellet were assayed for HCT activity, and profiled by SDS-PAGE (10\% resolving gel, $5 \%$ stacking gel) using Coomassie brilliant blue staining [36]. Negative controls used comparable preparations harbouring a void vector.

\section{Enzyme assay and the determination of kinetic parameters} CGA was purchased from Sigma-Aldrich (Germany), and quinic acid from Fluka (Switzerland). CoA esters (substrates) were kindly provided by Dr. Legrand (CNRS-IBMP laboratory Strasbourg, France) and then synthesised using the procedure proposed by Beuerle and Pichersky [41]. 4CL enzyme was kindly provided by Dr. Douglas (University of British Columbia Vancouver). The $20 \mu \mathrm{l}$ reaction mixture contained $100 \mathrm{mM}$ sodium phosphate buffer $(\mathrm{pH}$ 7.5), $1 \mathrm{mM}$ dithiothreitol, between $50 \mathrm{ng}$ and $1 \mu \mathrm{g}$ of enzyme, and the various substrates ( $p$-coumaroyl-CoA, caffeoyl-CoA, quinic acid and shikimic acid) at concentrations ranging from $0.1 \mathrm{mM}$ to $5 \mathrm{mM}$. The reverse reaction, 
i.e. conversion of chlorogenic acid and CoA into caffeoylCoA, was tested as follow: $0.8 \mu \mathrm{g}$ enzyme was incubated in presence of $1 \mathrm{mM}$ dithiothreitol, $100 \mu \mathrm{M}$ of chlorogenate and $100 \mu \mathrm{M}$ CoA. Reactions were initiated by enzyme addition, incubated at $30^{\circ} \mathrm{C}$ for $30 \mathrm{~min}$, and terminated by the addition of $20 \mu \mathrm{l}$ of acetonitrile/ $\mathrm{HCl}$ (99:1). Reaction products were analysed by reverse-phase HPLC on a C18 column (LiChroCART 125-4, Merck), using the pair of solvents $90 \% \mathrm{H}_{2} \mathrm{O}, 9.9 \% \mathrm{CH}_{3} \mathrm{CN}, 0.1 \% \mathrm{HCOOH}$ and $80 \% \mathrm{CH}_{3} \mathrm{CN}, 19.9 \% \mathrm{H}_{2} \mathrm{O}, 0.1 \% \mathrm{CH}_{3} \mathrm{COOH}$. The percentage of the latter reached the $60 \%$ over a 15 min run time, and $100 \%$ after $28 \mathrm{~min}$.

Varying substrate-dependent concentrations were used for $K_{\mathrm{m}}$ determination. These consisted of $0.8 \mu \mathrm{g}$ enzyme, 300 $\mu \mathrm{M} p$-coumaroyl-CoA and 0.1-30 mM quinate for $K_{\mathrm{m}}$ of quinate. $K_{\mathrm{m}}$ of $p$-coumaroyl-CoA was established with 10$400 \mu \mathrm{M} p$-coumaroyl-CoA with quinate $(20 \mathrm{mM})$ or $100-$ $600 \mu \mathrm{M}$ with shikimate $(20 \mathrm{mM})$ as acceptors and $0.8 \mu \mathrm{g}$ enzyme. For caffeoyl-CoA $K_{\mathrm{m}}$ determination, $0.8 \mu \mathrm{g}$ enzyme, $20 \mathrm{mM}$ quinate and $10-100 \mu \mathrm{M}$ caffeoyl-CoA were used. Reactions were incubated at $30^{\circ} \mathrm{C}$ for $10 \mathrm{~min}$. An HPLC calibration curve was established for each molecule for quantification purpose. $K_{\mathrm{m}}$ and $V_{\max }$ values were calculated from triplicated by Lineweaver-Burk plots.

\section{Extraction of polyphenolic compounds, HPLCIDAD and HPLCIMS analyses}

On the basis of a genetic diversity assessment of 118 globe artichoke accessions [2] about 30 entries were chosen to represent the genetic variation present. Leaves of these entries were assayed in triplicate for leaf polyphenolic composition using HPLC/DAD/MS. Mono- and di-caffeoylquinic derivatives, apigenin and luteolin glycosides were characterised and quantified. On the basis of these observations, two globe artichoke entries ('Romanesco C3' and 'Violet Margot'), one cultivated cardoon (accession '41') and one wild cardoon (accession 'Kamaryna') were carried forward for a detailed study of the correlation between leaf polyphenolic composition and HCT expression.

Leaf tissues from these four different plants at 22-23 fullyexpanded leaves stage were collected. Growth conditions and management practices were similar to those used in commercial cultivation. Half of the plant material has been freezed immediately in liquid nitrogen, whereas the other part was lyophylized within $12 \mathrm{~h}$ of collection and the dried materials were ground to a powder and stored in sealed bags at room temperature. The powders $(2 \mathrm{~g})$ were extracted in $70 \% \mathrm{v} / \mathrm{v}$ ethanol $(\mathrm{pH} 2)$, cleared through a $0.45 \mu \mathrm{m}$ filter, and the extractants first concentrated under vacuum (Rotavapor $144 \mathrm{R}$, Büchi, Switzerland) then rinsed with ultra pure acid water (MilliQ system, Waters Co., USA) adjusted to pH 2 with formic acid. Defatting was achieved by the addition of n-hexane, and the samples were re-concentrated under vacuum to a final volume of $25 \mathrm{ml}$. Lyophilised extracts were stored at $-20^{\circ} \mathrm{C}$.

Leaves polyphenolic composition were analysed using HPLC/DAD and HPLC/MS. HPLC/DAD analyses were performed via a HP-1100 liquid chromatograph equipped with a DAD detector and a HP 1100 MSD APIelectrospray (Agilent Technologies, USA). Polyphenol compounds were separated using a $150 \times 4.6 \mathrm{~mm}(5 \mu \mathrm{m})$ Luna RP18 (Phenomenex) maintained at $27^{\circ} \mathrm{C}$. The eluents were $\mathrm{H}_{2} \mathrm{O}$ ( $\mathrm{pH} 3.2$ adjusted with formic acid), and $\mathrm{CH}_{3} \mathrm{CN}$. A four-step linear solvent gradient system was used, starting from $0 \%$ and reaching $100 \% \mathrm{CH}_{3} \mathrm{CN}$ over a $30 \mathrm{~min}$ run, using a flow rate of $0.6 \mathrm{ml} / \mathrm{min}$. The percentage of $\mathrm{CH}_{3} \mathrm{CN}$ was $20 \%$ from $0-5 \mathrm{~min}, 30 \%$ from $7-13$ min, and $100 \%$ from $20-30 \mathrm{~min}$. UV-vis spectra were recorded in the range $190-600 \mathrm{~nm}$, and chromatograms were obtained at $330 \mathrm{~nm}$.

HPLC/MS analyses were performed using a HP 1100L liquid chromatograph linked to a HP 1100 MSD mass spectrometer with an API/ESI interface (Agilent Technologies, California, USA). The mass spectrometer operating conditions were: gas temperature, $350^{\circ} \mathrm{C}$; nitrogen flow rate, $10.5 \mathrm{~L} / \mathrm{min}$; nebulizer pressure, $40 \mathrm{psi}$; quadrupole temperature, $40^{\circ} \mathrm{C}$; and capillary voltage, $3500 \mathrm{~V}$. The orthogonal position of the nebulizer with respect to the capillary inlet enabled the use of the same conditions as for HPLCDAD analysis. The mass spectrometer was operated in negative mode; and the fragmentor was set at $120 \mathrm{eV}$ for the standard and the isolated compounds.

Individual polyphenols were identified by comparison and combination of their retention time, UV-Vis and mass spectra of the peaks with those of authentic samples or isolated compounds previously characterized [42]. Cynarin was obtained from Roth (Germany) and chlorogenic acid from Sigma-Aldrich (St. Louis, USA). Quantification of individual polyphenols was performed directly by HPLC-DAD using a four-point regression built with the available standards. Curves with a correlation factor above 0.9998 were considered to be significant. Quantification of caffeoylquinic mono- and di-esters was inferred from $330 \mathrm{~nm}$ chromatograms using CGA and cynarin for reference.

\section{Development of C. cardunculus probes and northern blot analysis}

For northern blot analyses, 3 probes were developed by applying a PCR Dig Probe Synthesis Kit (Roche Applied Science, Switzerland) with ART3For and ART3Rev primers (Table 1) designed from the C. cardunculus HCT sequence: probe 1 from globe artichoke, probe 2 from cultivated cardoon, and probe 3 from wild cardoon. 
RNA were extracted from the second freezed part of the plant material described above. Total RNA separated on formaldehyde-agarose gels [36] before blotting to a nylon membrane (Amersham Pharmacia Biotech, UK) via overnight capillarity transfer. RNAs quality was confirmed by ethidium bromide staining of the gel (Fig. 7, first panel) following electrophoresis. Furthermore, RNA analysis was normalized by using a constitutively expressed gene (RNA 18S, Fig. 7, second panel). Hybridization was performed at $50^{\circ} \mathrm{C}$ for $16 \mathrm{~h}$ with a denatured DNA probe. After hybridization, stringency washes $(2 \times 15 \mathrm{~min}$ at RT in $2 \times$ SSC, $0.1 \%$ SDS and $2 \times 15 \mathrm{~min}$ at $68^{\circ} \mathrm{C}$ in $0.1 \times$ SSC, $0.1 \%$ SDS) was performed. The membrane subsequently was incubated with anti-DIG-AP conjugate (diluted 1:20,000), and visualised using CDPStar reagents (Roche Applied Science, Switzerland) by a 15-60 min exposure to X-ray film.

\section{Authors' contributions}

SL and FB planned and supervised the work. CC and AH carried out the molecular genetic studies; EP and AA carried out the phylogenetic analysis; AR and RL performed the HPLC's. All authors read and approved the final manuscript.

\section{Acknowledgements}

We thank Prof. G. Mauromicale and Dr. R. Mauro of the University of Catania for providing plant material. We are particularly grateful to Dr. P. Pinelli for extraction of polyphenolic compounds and HPLC analyses. We are grateful to Dr. M. Legrand (CNRS-IBMP Strasbourg, France) and Dr. C.). Douglas (University of British Columbia Vancouver) for providing CoA esters substrates and $4 C L$ enzyme respectively. We thank Dr. P. Ullmann (CNRS-IBMP Strasbourg, France) for technical advices in the caffeoyl-CoA and $p$-coumaroyl-CoA synthesis. Thanks are also due to Dr. G.P. Accotto and Dr. G. Mason of the IVV-CNR of Torino for technical assistance in the northern blot analyses.

\section{References}

I. Rottenberg A, Zohary D, Nevo E: Isozyme relationships between cultivated artichoke and the wild relatives. Genetic Resources and Crop Evolution 1996, 43:59-62.

2. Lanteri S, Saba E, Cadinu M, Mallica GM, Baghino L, Portis E: Amplified fragment length polymorphism for genetic diversity assessment in globe artichoke. Theor Appl Genet 2004, 108: 1534-1544.

3. Acquadro A, Portis E, Lee D, Donini P, Lanteri S: Development and characterisation of microsatellite markers in Cynara cardunculus L. Genome 2005, 48:2 17-225.

4. Dogan S, Turan Y, Erturk H, Arslan O: Characterization and purification of polyphenol oxidase from Artichoke (Cynara scolymus L.). J Agric Food Chem 2005, 53:776-785.

5. Slanina J, Taborska E, Musil P: Determination of cynarine in the decoctions of the artichoke (Cynara cardunculus L.) by the HPLC method. Cesko-SloV Farm 1993, 42:265-268.

6. Wagenbreth D: Evaluation of artichoke cultivars for growing and pharmaceutical use. Beitr Zuchtungsforsch 1996, 2:400-403.

7. Sevcikova P, Glatz Z, Slanina J: Analysis of artichoke (Cynara cardunculus $L$.) extract by means of micellar electrokinetic capillary chromatography. Electrophoresis 2002, 23:249-252.

8. Wang MF, Simon JE, Aviles IF, He K, Zheng QY, Tadmor Y: Analysis of antioxidative phenolic compounds in artichoke (Cynara scolymus L.). J Agr Food Chem 2003, 5 1:60 I-608.

9. Gebhardt R: Antioxidative and protective properties of extracts from leaves of artichoke (Cynara scolymus L.) against hydroperoxide induced oxidative stress in cultured rat hepatocytes. Toxicol Appl Pharm 1997, 144:279-286.

10. Brown JE, Rice-Evans CA: Luteolin rich artichoke extract protects low density lipoprotein from oxidation in vitro. Free Radical Res 1998, 29:247-255.

II. Perez-Garcia F, Adzet T, Canigueral S: Activity of artichoke leaf extract on reactive oxygen species in human leukocytes. Free Radical Res 2000, 33:66I-665.

12. Kraft K: Artichoke leaf extract. Recent findings reflecting effects on lipid metabolism, liver and gastrointestinal tracts. Phytomedicine 1997, 4:369-378.

13. Pittlern MH, Ernst E: Artichoke leaf extract for serum cholesterol reduction. Perfusion 1998, I I:338-340.

14. Mcdougall B, King PJ, Wu BW, Hostomsky Z, Manfred G, Robinson WE: Dicaffeoylquinic acid and dicaffeoyltartaric acid are selective inhibitors of human immunodeficiency virus type I integrase. Antimicrob Agents Ch 1998, 42: 140-146.

15. Slanina J, Taborska E, Bochorakova H, Slaninova I, Humpa O, Robinson WE, Schram H: New and facile method of preparation of the anti-HIV-I agent, I,3-dicaffeoylquinic acid. Tetrahedron Lett 200I, 42:3383-3385.

16. Martino V, Caffini N, Phillipson JD, Lappa A, Tchernitchin A, Ferraro $\mathrm{G}$, Debenedelli S, Schilcher $\mathrm{H}$, Acevedo C: Identification and characterization of antimicrobial components in leaf extracts of globe artichoke (Cynara scolymus L.). Acta Horticulturae 1999, 50I:III-II4.

17. Zhu XF, Zhang HX, Lo R: Phenolic compounds of artichoke (Cynara scolymus $L$ ) and their antimicrobial activities. J Agric Food Chem 2004, 52:7272-7278.

18. Zhu XF, Zhang HX, Lo R: Antifungal activity of Cynara scolymus L. extracts. Fitoterapia 2005, 76: I08-III.

19. Winkel-Shirley B: Evidence for enzyme complexes in the phenylpropanoid and flavonoid pathways. Physiologia Plantarum 1999, 107:142-149.

20. Humphreys JM, Chapple C: Rewriting the lignin roadmap. Current Opinion in Plant Biology 2002, 5:224-229.

2I. Hoffmann L, Maury S, Martz F, Geoffroy P, Legrand M: Purification, cloning and properties of an acyltransferase controlling shikimate and quinate ester intermediates in phenylpropanoid metabolism. The journal of biological chemistry 2003, 278:95-103.

22. St Pierre B, Laflamme P, Alarco AM, De Luca V: The terminal Oacetyltransferase involved in vindoline Biosynthesis defines a new class of proteins responsible for Coenzyme $A$ dependent acyl transfer. Plant 」 1998, 14:703-7|3.

23. Brown NF, Anderson RC, Caplan SL, Foster DW, McGarry JD: Catalytically important domains of rat carnitine palmitoyltransferase II as determined by site-directed mutagenesis and chemical modification. Evidence for a critical histidine residue. J Biol Chem 1994, 269:19157-19162.

24. Suzuki H, Nakayama T, Nishino T: Proposed mechanism and functional amino acid residues of Malonyl-CoA: Anthocyanin 5-O-Glucoside-6"'-O-Malonyltransferase from flowers of Salvia splendens, a member of the Versatile Plant Acyltransferase Family. Biochemistry 2003, 42: 1764-I77I.

25. Niggeweg R, Michael AJ, Martin C: Engineering plants with increased levels of the antioxidant chlorogenic acid. Nature biotechnology 2004, 22:746-754.

26. Rhodes MJC, Wooltorton LSC: The enzymic conversion of hydroxycinnamic acids to $p$-coumarylquinic and chlorogenic acids in tomato fruits. Phytochemistry 1976, 15:947-95 I.

27. Ulbrich B, Zenk MH: Partial purification and properties of hydroxycinnamoyl-CoA: quinate hydroxycinnamoyl transferase from higher plants. Phytochemistry 1979, 18:929-933.

28. Harborne JB: Classes and functions of secondary products from plants. In Chemicals from plants Edited by: Walton NJ, Brown DE. Imperial College Press: World Scientific; 1999: I-25.

29. Villegas RA, Kojima M: Purification and characterization of Hydroxycinnamoyl D-Glucose: quinate hydroxycinnamoyl transferase in the root of sweet potato, ipomoea batatas lam. The journal of biological chemistry 1986, 26 I:8729-8733.

30. Schoch G, Goepfert S, Morant M, Hehn A, Meyer D, Ullmann P, Werck-Reichhart D: CYP98A3 from Arabidopsis thaliana is a 3'hydroxylase of phenolic esters, a missing link in the phenylpropanoid pathway. The journal of biological chemistry 200I, 276:36566-36574. 
31. Franke R, Humphreys JM, Hemm MR, Denault JW, Rueggerß MO, Cusumano JC, Chapple C: The Arabidopsis REF8 gene encodes the 3-hydroxylase of phenylpropanoid metabolism. The Plant Journal 2002, 30:33-45.

32. Morant M, Bak S, Moller BL, Werck-Reichhart D: Plant cytochromes P450: tools for pharmacology, plant protection and phytoremediation. Current opinion in biotechnology 2003, 14:15I-162.

33. Burhenne K, Kristensen BK, Rasmussen SK: A New Class of $\mathbf{N}$ Hydroxycinnamoyltransferases. Purification, cloning, and expression of a barley agmatine coumaroyltransferase (ec 2.3.1.64). The journal of biological chemistry 2003, 278:13919-13927.

34. Hoffmann L, Besseau S, Geoffroy P, Ritzenthaler C, Meyer D, Lapierre C, Pollet B, Legrand M: Silencing of hydroxycinnamoyl-coenzyme A shikimate/quinate hydroxycinnamoyltransferase affects phenylpropanoid biosynthesis. Plant Cell 2004, 16:1446-1465.

35. Strack D, Gross W: Properties and activity changes of chlorogenic acid-glucaric acid caffeoyltransferase from tomato (Lycopersicon esculentum). Plant Physiol 1990, 92:4I-47.

36. Sambrook J, Russell DW: Molecular Cloning 3rd edition. New York: CSHL Press; 2001

37. Morant M, Hehn A, Werck-Reichhart D: Conservation and diversity of gene families explored using the CODEHOP strategy in higher plants. BMC Plant Biol 2002, 2:7.

38. BMR genoics [http://www.bmr.cribi.unipd.it]

39. ClustalW [http://www.ebi.ac.uk/clustalw]

40. Kumar S, Tamura K, Nei M: MEGA3: Integrated software for Molecular Evolutionary Genetics Analysis and sequence alignment. Briefings in Bioinformatics 2004, 5: 150-163.

41. Beuerle T, Pichersky E: Enzymatic Synthesis and Purification of Aromatic Coenzyme A Esters. Analytical Biochemistry 2002, 302:305-3। 2.

42. Romani A, Pinelli P, Cantini C, Cimato A, Heimler D: Characterization of Violetto di Toscana, a Italian variety of artichoke (Cynara scolymus L.). Food Chemistry 2006, 95:221-225.

Publish with Bio Med Central and every scientist can read your work free of charge

"BioMed Central will be the most significant development for disseminating the results of biomedical research in our lifetime. "

Sir Paul Nurse, Cancer Research UK

Your research papers will be:

- available free of charge to the entire biomedical community

- peer reviewed and published immediately upon acceptance

- cited in PubMed and archived on PubMed Central

- yours - you keep the copyright

Submit your manuscript here:

http://www.biomedcentral.com/info/publishing_adv.asp
BioMedcentral 